\title{
Primer on the Pathogenesis of Severe COVID-19: Part One
}

Our Editor's Pick for this EMJ flagship issue is a two-part review by Walsh that discusses the pathogenesis of coronavirus disease (COVID-19) by firstly providing a breakdown of the complex cellular host-viral interactions, before discussing the key mediators of specific

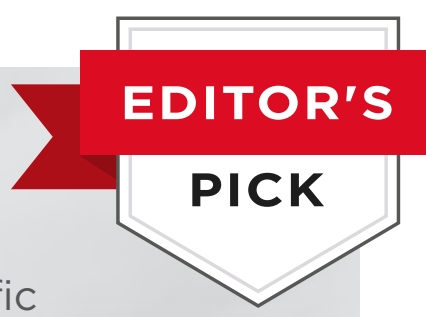
pathogenicity. With the search for a vaccine remaining top of the agenda worldwide, an increased understanding of the pathophysiology of this disease is essential in guiding the therapeutic approaches being put in place to stem the spread of this pandemic. We hope that you enjoy reading this timely review.
Authors:
Thomas J. Walsh
Rheumatology Department, Harrogate and District Hospital, Harrogate, UK *Correspondence to tomjwalsh81@gmail.com

Disclosure: The author has declared no conflicts of interest.

Received: 23.06.20

Accepted: 27.08.20

Keywords:

Cluster of differentiation 147 (CD147), coronavirus disease (COVID-19), NRLP3 Inflammasome, pneumonia, protein kinase $\mathrm{R}$, severe acute respiratory syndrome (SARS), severe acute respiratory syndrome coronavirus-2 (SARS-CoV-2).

Citation:

\section{Abstract}

In Part One of this exploration of the pathogenesis of coronavirus disease (COVID-19), the author will evaluate the viral and cellular immunological basis for the condition. The virus demonstrates a remarkable capability not just to evade, but to exploit host immune characteristics to perpetuate viral replication. In this regard, severe acute respiratory syndrome (SARS)/severe acute respiratory syndrome coronavirus-2 (SARS-CoV-2) disables most antiviral mechanisms, including the early interferon response, and avoids detection to permit unimpeded viral multiplication. Consequently, antigen-presenting cells fail to adequately stimulate the T-cell receptor. As a consequence, T-cell p53 remains highly expressed, which in turn disables an adequate effector T-cell response.

Replicating SARS-CoV-2 double-strand RNA robustly activates protein kinase R (PKR)/PKRlike endoplasmic reticulum kinase (PERK). While the virus is grossly invulnerable to its antiviral effects, PKR is crucial for effecting the cytokine milieu in COVID-19. PERK is a component of the unfolded protein response, which eventuates in autophagy. SARS virions use double-membrane vesicles and adapt PERK signalling not only to avoid autophagy, but to facilitate replication. Viral activation of PKR/PERK is mutually exclusive to NLRP3 stimulation. The NLRP3 pathway elaborates IL-1B. This is chiefly a feature of paediatric SARS/SARS-CoV-2 cases. The difficulties encountered in predicting outcome and forging effective therapeutics speaks to the breadth of complexity of the immunopathogenesis of this virus. 


\section{INTRODUCTION}

Severe and fatal severe acute respiratory syndrome coronavirus-2 (SARS-CoV-2) infection is characterised by chiefly pulmonary manifestations. Clinically, pneumonias have been subdivided into specific phenotypes: a spectrum from patchy ground-glass opacification to the oedematous lung with atypical acute respiratory distress syndrome features. ${ }^{1}$ Bilateral diffuse alveolar damage with cellular fibromyxoid exudates and desquamation of pneumocytes with hyaline membrane formation are pathologically apparent. ${ }^{2}$ For the majority of patients (varying by age and other factors), the condition has been mild. It appears that the causative virus, SARS-CoV-2, evolved into two major genomic types ( $L$ and $S$ types), with a roughly 70:30 split. ${ }^{3}$ Although the L-type is likely phenotypically more aggressive, variability in host response is clearly a major determinant of outcome. Studies of SARSCoV-2 have been limited by the novel nature of the virus. Nonetheless, valuable insight may be drawn from existing studies on the biology and pathogenesis of the SARS virus because of the significant sequence homology. ${ }^{4}$

\section{VIRUS-SPECIFIC CHARACTERISTICS: ESTABLISHING INFECTION}

Extrapolating from SARS-CoV virology, initial investigations implied that SARS-CoV-2 used angiotensin-converting enzyme 2 (ACE2) for cellular entry. ${ }^{5}$ ACE2 expression is predominantly, but not limited to, pulmonary tissue (Type 2 pneumocytes and ciliated airway epithelial cells), with expression of SARS virus previously reported in extrapulmonary ACE2+ tissues. ${ }^{6-9}$ SARS spike proteins (S-proteins) were found to bind robustly to ACE2. ${ }^{10,11}$ Recent reports suggest that the nature of SARS-CoV-2 affinity for ACE2 is 10-times more avid than that of SARS. TMPRSS2, a serine protease, is used for S-protein priming within cells, which is essential for viral spread and (especially pulmonary) pathogenesis in the infected host (cathepsins B and L may also be used but are inessential for this purpose). ${ }^{5,12} \mathrm{~A}$ further portal of entry is cell surface expression of cluster of differentiation 147 (CD147), also known as basigin or extracellular matrix metalloproteinase inducer (EMMPRIN). ${ }^{13-15}$
Of the large coronaviral genome, the open reading frames $1 \mathrm{a}$ and $1 \mathrm{~b}$ (ORF1a and $1 \mathrm{~b}$ ), encoding the viral replicase, comprise twothirds. The replicase includes multispanning transmembrane proteins that physically anchor the replication/transcription complex to the intracellular membranes. Within the cytoplasm, double-membrane vesicles (DMV) branch off from the rough endoplasmic reticulum (ER), reminiscent of autophagosomes. ${ }^{16}$ The coronavirus endonuclease, which resides in the replication complex, prevents simultaneous activation of double-strand RNA (dsRNA) sensors melanoma differentiation-associated protein 5, 2'-5'-oligoadenylate synthetase 3, and protein kinase $R(P K R)$. This strategy permits evasion of the host innate antiviral defenses. ${ }^{17}$ Thus, viral kinetics are rapid in the early period (first 48 hours) post-infection.

\section{THE IMMUNOLOGICAL RESPONSE IN SEVERE COVID-19 INFECTION}

Severe SARS/SARS-CoV-2 is suggestive of an apparent biphasic (dysregulated) immune response. ${ }^{18}$ A weak or absent interferon Type 1 ([IFN-1] i.e., IFNa and IFNB) response during the early phase of SARS-CoV-2 infection plays an important role in permitting viral replication within nasopharyngeal cells and pneumocytes. $^{19,20}$ It is apparent that virion nonstructural protein 1 within infected cells can suppress host gene expression (including IFN-1), promote host messenger RNA (mRNA) degradation, and inhibit host protein translation. ${ }^{21}$ Furthermore, the SARS coronavirus papain-like protease induces inhibition of the production of IFN-1 and proinflammatory cytokines in toll-like receptor 3 (TLR3), retinoic acid-inducible gene 1 , and TLR7 signalling pathways, thus disabling detection mechanisms in the endosome and in the cytoplasm. . $2-26^{2}$

Indeed, some investigators could find no or only modest evidence of IFN response to SARS infection. ${ }^{27,28}$ However, fatal/severe SARS in humans was accompanied by a late but robust and persistent expression of IFN-1, especially from plasmacytoid dendritic cells. Concurrent expression of IFN-1 and IFN-stimulated genes appear to preclude adequate T-cell and antibody responses; ;8,29-31 this was demonstrated in SARSinfected IFNaß receptor knockout (IFNAR ${ }^{-/}$) 
mice, who exhibited only mild to moderate weight loss and clinical disease. These mice demonstrated minimal alveolar oedema and increased peribronchiolar/perivascular immune cell infiltration, which resolved with viral clearance by 10 days post-infection. By contrast, BALB/c mice with intact IFNAR exhibited a sixfold increase in (mainly) inflammatory monocytemacrophages by Day 3 post-infection, which is too late for the peak of viral replication. This was then abrogated in the absence of IFN-1.32

Augmented T-cell apoptosis in SARS infection impedes T-cell response and engenders a relative Iymphopaenia. ${ }^{33}$ Normally, IL-2 promotes the differentiation of effector $T$ cells in the presence of antigen-specific T-cell receptor (TCR) stimulation. TCR engagement by peptidebound major histocompatability complex (MHC) molecules on antigen-presenting cells (APC) changes the topology of the TCR, inducing the formation of microclusters at the $T$ cell-APC interface, named the immunological synapse. This arrangement helps to improve T-cell antigen recognition. ${ }^{34}$

However, in SARS, antigen-specific TCR expression is weakened by an early hyporesponsiveness of APC, especially in dendritic cells. SARS-CoV does not upregulate the expression of CD83, CD86, MHC Class I, or MHC Class II molecules on immature dendritic cells. ${ }^{35}$ Also, the late surge in IFN $\beta$ alters histone modifications in the IL-2 promoter to retain the locus in an inaccessible configuration, thereby curbing the T-cell response. ${ }^{36}$ Any elevation in IL-2 expression, secondary to immune monocytemacrophage stimulation of naïve $T$ cells, induces a sustained increase in p53 protein expression in the $T$ cell. Downmodulation of p53 is necessary for antigen-specific responses of naïve and antigen-primed peripheral $T$ cells and T-cell clones. This prevents proliferative TCR signalling, critical for antigen-specific CD4(+) T-cell responses, despite adequate signalling through the IL-2 receptor. ${ }^{37}$ It is this failure to recruit CD4+ $T$ cells to the lungs (and not CD8+ $T$ cells) that adversely affects viral clearance. The absence of the CD4+ T-cell response leads to enhanced interstitial pneumonitis. ${ }^{18}$ Furthermore, respiratory tract memory CD4+ T cells, in conjunction with IFNy, would provide a more robust intermediateterm defence against reinfection. ${ }^{38}$ The latter may account for the inconsistent nature of postinfective antibody production.
$T$ cells can attenuate cytokine storms by suppressing the immune response. ${ }^{39}$ It is the impairment of the regulatory mechanisms of $T$ cells in a cytokine-rich milieu that fosters the development of the cytokine storm. ${ }^{40}$

In view of the abortive nature of T-cell infection by SARS-CoV-2, it is unlikely that this, per se, makes a significant contribution to the observed lymphopenia in coronavirus disease (COVID-19) infections. ${ }^{41}$

\section{DISEASE-SPECIFIC IMMUNOLOGY}

\section{The Role of Protein Kinase $\mathrm{R}$}

While the impairment of host gene expression undoubtedly plays a part in infected epithelial cell apoptosis, much of the apoptosis appears to be secondary to host PKR expression, which is engaged as a stratagem to detect replicating dsRNA. PKR induces phosphorylation of elongation initiation factor $2 a$ (elF2a) which, in turn, blocks protein synthesis through translation of mRNA. The phosphorylated elF2a also encodes for antiviral factors and mediates the integrated stress response. ${ }^{41}$ The blockade of protein synthesis results in the decrease or prevention of viral replication, and may result in apoptosis. ${ }^{42}$ PKR can also induce apoptosis independently of elF2a phosphorylation, by activation of the FAS-associated death domain (FADD)/caspase-8/caspase-3, and caspase-9 apoptotic protease-activating factor-1 (APAF-1) pathways. ${ }^{43-46}$ In spite of a significant burden of PKR expression, SARS-CoV is not susceptible to its antiviral activities. Indeed, rather than inhibiting PKR activation, translation of SARS-CoV mRNA proceeds despite elF2a phosphorylation. ${ }^{42}$

PKR increases IFN1 expression via an elF2aindependent mechanism by regulating IFNa/ $\beta$ mRNA stability. ${ }^{41,47}$ As well as contributing to the development of interstitial pneumonia, this spike in IFN1 creates an autoamplifying loop, given that IFN1 is known to enhance PKR expression. ${ }^{42}$ Activation of PKR by dsRNA has been shown in several cell types, including airway epithelial cells, to result in phosphorylation of $\mathrm{IKB}$ and therefore activation of NF-kB. ${ }^{48-51}$ p38 mitogen-activated protein kinase (MAPK) expression requires PKR. ${ }^{52,53}$ dsRNA induction of TNF-a requires PKR activation, but IL-1 $\beta$ induction follows a PKRindependent pathway. ${ }^{54} \mathrm{IL}-6, \mathrm{IL}-8$, and Regulated upon Activation, normal T-cell expressed and 
presumably secreted (RANTES), among others, are promoted by PKR expression. ${ }^{42}$ Exuberant induction of PKR is the substantial driving force behind the cytokine-rich milieu observed in SARS/SARS-CoV-2 infection (Figure 1).

Note should be made here that levels of PKR may be already elevated chronically in individuals by virtue of a string of host factors including obesity, ageing, diabetes, congestive heart failure, cancer, and genetic factors (e.g., ApoE4, the most prevalent risk factor for Alzeihmer's disease), all of which have been found to yield worse outcomes in SARS-CoV-2 infections. ${ }^{55,56}$

\section{The Role of NLRP3 Inflammasome (Paediatrics)}

Paediatric SARS patients were demonstrated to have markedly elevated circulating IL-1 This suggests selective activation of a caspase1-dependent pathway. Notably, only mild/ minimal activation of IL- 6 and TNF-a was noted in paediatric populations. ${ }^{57}$ However, as indicated above, IL-1B is independent of the PKR pathway. Rather, IL-1B is a caspase cleavage product of the nucleotide-binding domain and leucine-rich repeat and NLRP3 pathway. ${ }^{58}$ It appears that the SARS-COV ORF8b robustly activates the NLRP3 inflammasome by providing a potent signal 2, required for activation. Note that inflammasome activity requires signals to effect cleavage: signal 1 is a priming signal and signal 2 is an activation signal. Mechanistically, ORF8b interacts directly with the leucine-rich repeat domain of NLRP3 and localises with NLRP3 and apoptosis-associated speck-like protein containing a caspase recruitment domain (ASC) in cytosolic dot-like structures. ORF8b triggers cell death consistent with pyroptotic cell death in macrophages; while in those cells lacking NLRP3, accumulating ORF8b cytosolic aggregates cause ER stress, mitochondrial dysfunction, and caspase-independent cell death. ${ }^{59}$ Likewise, SARS-CoV ORF3a protein activates the NLRP3 inflammasome by promoting TRAF3-dependent ubiquitination of ASC. ${ }^{60}$

It has been shown that PKR can suppress the NLRP3 inflammasome activation by modulating the microtubular cytoskeleton. ${ }^{61,62}$ It is evident, therefore, that a key mediator of the differential clinical presentation apparent between the paediatric population (who almost universally experience a mild or at worst moderate infection) and older adults (especially those with the aforementioned comorbidities, who are more predisposed to severe illness with COVID-19) is whether or not the NLRP3 pathway is activated in preference to a mutually exclusive pathway involving PKR overexpression.

The relatively rare occurrence of the Kawasakilike syndrome as part of the spectrum of paediatric presentation with COVID-19, known as paediatric inflammatory multisystem syndrome temporally associated with SARS-CoV-2 infection in Europe or multisystem inflammatory syndrome in children in the USA, bears the hallmarks of an autoinflammatory syndrome/vasculitis, whose pathogenesis owes to IL-1ß overexpression. ${ }^{63,64}$

\section{The Role of Protein Kinase R Endoplasmic Reticulum Kinase}

PERK is also elaborated as a consequence of dsRNA presence within the cytoplasm. PERK is a sensor in the unfolded protein response pathway. ${ }^{65}$ PERK is further activated by the SARS-CoV 3a protein. As well as causing elF2a phosphorylation, it stimulates expression of ER molecular chaperones such as glucose-regulated protein 78 (GRP78) and GRP94. ${ }^{66}$ Increased transcriptional activation and biosynthesis of ER chaperones would enhance folding of the 3a and other viral proteins in the ER lumen. ERassociated degradation (ERAD) will normally delete an increased load of unfolded and misfolded proteins within the ER. ${ }^{67}$ This requires activation of the Inositol-requiring enzyme-1/Xbox binding protein-1 and activating transcription factor 6 pathways. However, by activating PERK only, and not these ERAD components, the SARS-CoV 3 a protein is able to enhance folding of common viral structural proteins S, E, M, and $\mathrm{N}$, while avoiding activation of ERAD (which would be detrimental to virion assembly and likely trigger autophagy-dependent cell death). ${ }^{68}$

SARS-CoV must traffic across the ER membrane, thereby forming structures called doublemembrane vesicles (DMV), which are thought to provide the necessary platform for the viral replication process while avoiding immune detection. ${ }^{69}$ DMV, which originate from the ER membrane, contain nonstructural transmembrane proteins (nsp)3 and nsp4 and viral dsRNA, ${ }^{70,71}$ but lack markers typical for the ER Golgi intermediate compartment or Golgi. ${ }^{72}$ 


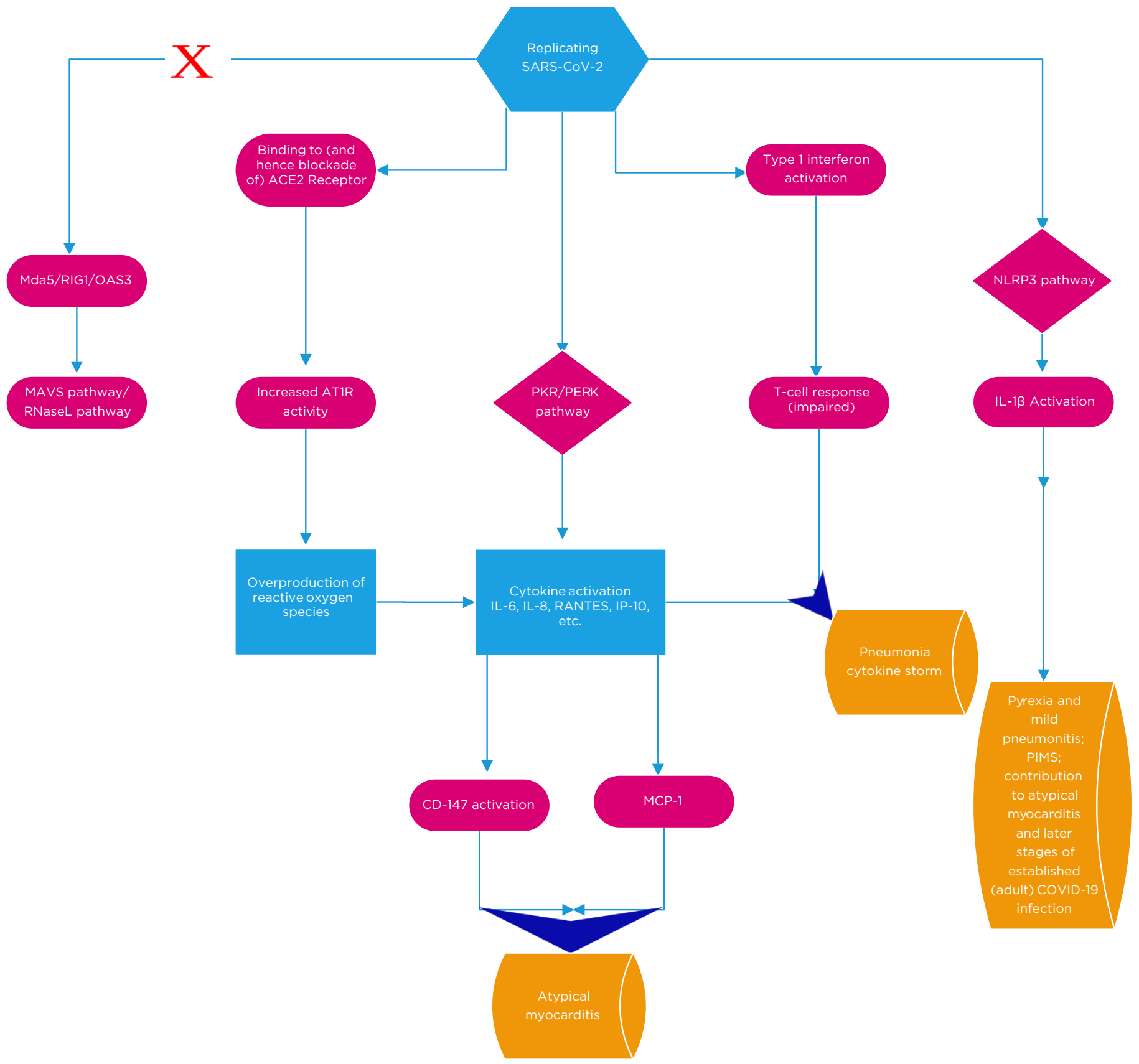

Figure 1: Overview of the basic cellular mechanisms contributing to coronavirus disease (COVID-19) pathogenesis.

Viral S protein binding to ACE2 receptors results in preferential activation of AT1R. The net consequence of this is a local overexpression of reactive oxygen species. In adults, the PKR/PERK pathways are preferentially activated to the exclusion of other antiviral pathways. This leads to the upsurge incytokine production. Subsequent consequences of this include activation of CD147 and MCP1, which contribute to atypical myocarditis. SARS CoV-2 initially disables the Type 1 interferon response but, subsequent to peak viraemia, Type 1 interferons are produced to excess. This impairs $\mathrm{T}$ cell response. The pro oxidant milieu in conjunction with the cytokine excess and blunted $\mathrm{T}$ cell response culminate in COVID-19 pneumonia and cytokine storm (across a spectrum of clinical severity). The NLRP3 pathway appears to be active early in paediatric infections to the exclusion of PKR/PERK (and at a later stage in adult disease). The clinical consequences include a relatively milder pneumonitis and pyrexia and rarely, in extreme circumstances, PIMS. It also contributes to the later stages of infection in adults.

ACE2: angiotensin converting enzyme 2; AT1R: angiotensin 1 receptor; R/G1: retinoic acid inducible gene I; MAVS: mitochondrial antiviral signaling protein; MCP1: monocyte chemoattractant protein 1; Mda5: melanoma differentiation associated protein 5; OAS3: oligoadenylate synthetase; PERK: protein kinase R endoplasmic reticulum kinase; PIMS: paediatric multisystem inflammatory syndrome; PKR: protein kinase R; RANTES; regulated upon activation, normal $T$ cell expressed and presumably secreted; RNaseL: ribonuclease L; SARS-CoV-2: severe acute respiratory syndrome coronavirus 2. 
DMV are coated with microtubule-associated protein light chain 3 (LC3), which is a ubiquitinlike modifier. $^{73}$ These ubiquitin-like modifiers recognise specific receptors that target associated vesicles to particular cellular locations. ${ }^{71,72}$ LC3 can exist in in a lipidated (LC3II) or a nonlipidated form (LC3-I) form. LC3II is involved in fusion of autophagosomes to lysosomes/proteomes, ${ }^{74}$ but coronavirus DMV display the nonlipidated LC3-I and thereby evade destruction. ${ }^{11}$

In overview, it is clear that the PKR/PERK pathway is used for propagation of the viral lifecycle and is central to the cytokine-driven pathogenesis of the disease. This acknowledgement is not an assertion that PKR/PERK is the only pathway active in severe COVID-19 in adult patients. SARS-CoV-2 is a positive-sense single-stranded RNA virus that activates PKR/PERK during the replicative process when dsRNA is present. When viral replication is reduced, the NLRP3 inflammasome can become active, particularly in later stages of infection. In addition, the NLRP3 caspase cleavage product, IL-1B, may be elaborated via noninflammasome means (e.g., during a protracted inflammatory process via release of neutrophil serine proteases).

\section{THE CONTRIBUTION OF CD147 TO SEVERE DISEASE PATHOGENESIS}

Further to the exploitation of the PKR/PERK pathway, SARS-CoV-2 may also use the inflammatory milieu as a means to further its own cell invasive/replicative potential. For instance, IL- 6 has previously been demonstrated to promote expression of CD147, a key receptor for viral cellular entry. ${ }^{75-77}$ This may be a normally adaptive process in the host since its expression may, in conjunction with cyclophilins, contribute to the recruitment of immune cells to sites of inflammation via chemokine-like activity. ${ }^{78}$ Cyclophilins have previously been found to contribute to coronavirus pathology. Indeed, overexpression of SARS-CoV nonstructural protein 1, as well as infection with live SARSCoV, strongly increased signalling through the calcineurin/nuclear factor of activated $T$ cells (NFAT) pathway and enhanced the induction of IL-2. The latter is compatible with the immunopathogenicity and cytokine dysregulation observed in severe SARS cases. ${ }^{79}$
CD147/EMMPRIN is a widely expressed integral plasma membrane glycoprotein that is expressed at varying levels in many cell types, including haematopoietic, epithelial, and endothelial cells. ${ }^{80-82}$ Its cell surface expression (with cyclophilin cofactors) has been associated with interactions with extracellular matrix proteins, such as matrix metalloproteins, and integrins. $^{83,84}$ While it is appreciated that IL-6 alone can be responsible for induction of thromboinflammation, it appears that CD147 overexpression is also proficient in this role, eliciting leukocyte chemotaxis and adhesion, as well as platelet activation and subsequent thrombus formation through the binding of various interaction partners. ${ }^{85-87}$ CD147 may assist IL-6 with the intravascular expression of vitronectin, plasminogen activator inhibitor-1, and von Willebrand factor, which forms the prothrombotic phenotype in severe SARS cases. ${ }^{88,89}$

\section{THE VIRAL INDUCTION OF OXIDATIVE DAMAGE}

The SARS-CoV-2 spike protein binds with great avidity to the ACE2 receptor. ${ }^{12}$ Once the protein has been cleaved by the serine protease TMPRSS2, the virus can be endocytosed, likely via micropinocytosis. ${ }^{90}$ Another serine protease, ADAM-17, is activated upon binding of SARSCoV to ACE2 and facilitates viral entry. In fact, knockdown of ADAM-17 by small interfering RNA severely attenuates SARS-CoV cellular entry. ADAM-17 functions as a TNF-a cleavage enzyme and thus it contributes to the presence of TNF-a in SARS pneumonia. ${ }^{91}$ Pulmonary endothelial expression of ACE2 is lower in older adults relative to younger individuals. ${ }^{92}$ This would appear to imply that older adults should be less susceptible to contracting the virus (at least at a cellular, biological level) and has also served as something of a paradox in that patients with chronic obstructive pulmonary disease who actively smoke have higher levels of ACE2, but have had relatively low mortality compared to those with cardiovascular disease who tend to have relatively low expression of ACE2. ${ }^{93,94}$ Angiotensin II levels have been demonstrably high in patients with COVID-19, thus apparently vindicating the theory that viral sequestration of ACE2 has a biochemical consequence 
for the host. ${ }^{95}$ In spite of this, patients who contract COVID-19 are not rendered overtly hypertensive. Moreover, the renin-angiotensin system is activated in almost any physiological stress scenario. ${ }^{96}$

In the absence of ACE2, signalling via the angiotensin I receptor is enhanced, apparently contributing to lung injury and pulmonary oedema in SARS. ${ }^{97,98}$ Angiotensin II binding to the angiotensin I receptor mediates its adverse effect on the lung through various subtypes of NADPH oxidase to produce reactive oxygen species. ${ }^{99}$ Furthermore, NADPH oxidase enhances phosphorylation, and hence activation, of PKR. ${ }^{100}$ The specific pathogenic contribution of ACE2 sequestration to COVID-19 pneumonia has been reviewed elsewhere. ${ }^{101}$

Haem oxygenase-1 (HO-1) is a stress-inducible, anti-inflammatory, and cytoprotective enzyme expressed in most cell types in organisms. Under several stress stimuli, $\mathrm{HO}-1$ expression and activity is upregulated to catalyse the ratelimiting enzymatic step of haem degradation into carbon monoxide, free iron, and biliverdin. ${ }^{102}$ Besides its effects on cell metabolism, $\mathrm{HO}-1$ is also capable of modulating host innate and adaptive immune response to sepsis, transplantation, and autoimmunity, and prevents oxidative damage associated with inflammation. HO-1 can exert a significant antiviral activity against a wide variety of viruses. ${ }^{103}$ Its activation decreases the migration of polymorphonuclear leukocytes to the lung. This may, in certain circumstances, reduce oxidative tissue damage. ${ }^{104}$ Crucially, it has been demonstrated to inhibit dendritic cell activation and immunogenicity. ${ }^{105}$ Failure to recruit functional respiratory dendritic cells to the lungs has been identified as a key defect, permitting the pathogenesis of SARS-CoV-induced pneumonia. ${ }^{106,107}$ This may be pertinent in the early stages of SARS/SARS-CoV-2 pneumonia.

In myeloid cells, HO-1 forms a complex with interferon regulatory transcription factor 3 (IRF3), which is required for IRF3 target genes and alters responses in infected cells. ${ }^{106}$ In fact, HO-1-deficient macrophages will show reduced expression of IFN- $\beta$ and IRF3. SARS coronavirus papain-like protease inhibits IRF3 activation at a step after phosphorylation, which is dependent on the deubiquitination activity of papain- like protease. ${ }^{109}$ In later stages of the infection, caveolin-1 overexpression in lung epithelial cells will competitively inhibit $\mathrm{HO}-1$ (caveolin-1 will be discussed further in Part Two). ${ }^{110}$

\section{FUTURE DIRECTIONS: USING PATHOGENESIS TO BUILD DRUG PLATFORMS}

The PKR/PERK pathway is the pathogenic motive force behind the severity of COVID-19. The NLRP3 inflammasome fuels an alternative, mutually exclusive, and apparently more benign condition. It follows that blockade of the PKR/ PERK pathway would be distinctly advantageous to the host. While the expectation would be that it would not prevent infection, the nature of the infection would be much less severe.

To date, several pharmacological inhibitors of PKR have been investigated with varying degrees of adequacy. It is clear that identification of an inhibitor of PKR with good drug properties is an urgent necessity. There are some useful articles assessing this specific topic. ${ }^{111-114} \mathrm{~N}$-acetylcysteine has been demonstrated to partially remediate the apoptotic consequence of PKR activation and oxidative stress. ${ }^{100}$ Furthermore, $\mathrm{N}$-acetylcysteine has been demonstrated to alleviate the cytokine overproduction that occurs in alveolar Type II cells in the context of other respiratory viral infections such as influenza $A$ and $B$ and respiratory syncytial virus. ${ }^{115} \quad \mathrm{~N}$-acetylcysteine accomplishes this through inhibition of NF-kB translocation to the nucleus and phosphorylation of p38 MAPK, both of which are part of a stress pathway induced by PKR to promote IL-6, IL-8, and monocyte chemoattractant protein 1 overproduction. ${ }^{115-118}$

Pharmacological inhibitors of CD147 are also being developed for heterogenous indications. ${ }^{19-120}$ There may also be some rationale behind use of the humanised anti-CD147 IgG2 monoclonal antibody, meplazumab, which has been licensed as an orphan drug by the U.S. Food and Drug Administration (FDA) for treatment of malaria to counter not just viral entry into cells, but also the deleterious effects induced by CD147 in severe COVID-19.121 However, pharmacological evaluation is beyond the scope of this paper. 
systemic pathogenicity in COVID-19. It is the author's strong recommendation that the details

In conclusion, the author offers a rational breakdown of the cellular host-viral interaction in COVID-19. A substantial component of this analysis is extrapolated from the laboratory work already done in the field of SARS-CoV and other coronaviral infection/pneumonia. In Part Two, the author will probe the key mediators of specific presented herewith are made subject to scrutiny in detailed animal studies involving SARS-CoV-2. Furthermore, the investigation of the platforms for drug treatment require accelerated human trials to establish efficacy and to curb the ongoing toll of lives lost and economic damage caused by this pandemic.

\section{References}

1. Gattinoni L et al. COVID-19 pneumonia: different respiratory treatments for different phenotypes? Intensive Care Med. 2020;46(6):1099-102.

2. $X u Z$ et al. Pathological findings of COVID-19 associated with acute respiratory distress syndrome. Lancet Respir Med. 2020;8(4):420-2.

3. Tang $X$ et al. On the origin and continuing evolution of SARSCoV-2. National Science Review. 2020;7(6):1012-23.

4. Grifoni A et al. A sequence homology and bioinformatic approach can predict candidate targets for immune responses to SARS-CoV-2. Cell Host Microbe. 2020;27(4):671-80.

5. Hoffmann M et al. SARS-CoV-2 cell entry depends on ACE2 and TMPRSS2 and is blocked by a clinically proven protease inhibitor. Cell. 2020;181(2):271-80.

6. Sims AC et al. Severe acute respiratory syndrome coronavirus infection of human ciliated airway epithelia: role of ciliated cells in viral spread in the conducting airways of the lungs. J Virol. 2005;79(24):15511-24.

7. Hamming l et al. Tissue distribution of ACE2 protein, the functional receptor for SARS coronavirus. A first step in understanding SARS pathogenesis. J Pathol. 2004;203(2):631-7.

8. Ding $Y$ et al. Organ distribution of severe acute respiratory syndrome (SARS) associated coronavirus (SARS-CoV) in SARS patients: implications for pathogenesis and virus transmission pathways. J Pathol. 2004;203(2):622-30.

9. $\mathrm{Gu} J$ et al. Multiple organ infection and the pathogenesis of SARS. J Exp Med. 2005;202(3):415-24

10. Nie $Y$ et al. Highly infectious SARS-CoV pseudotyped virus reveals the cell tropism and its correlation with receptor expression. Biochem Biophys Res Commun. 2004;321(4):994-1000.

11. Glowacka I et al. Differential downregulation of ACE2 by the spike proteins of severe acute respiratory syndrome coronavirus and human coronavirus NL63. J Virol. 2010;84(2):1198-205.

12. Iwata-Yoshikawa $\mathrm{N}$ et al. tmprss 2 contributes to virus spread and immunopathology in the airways of murine models after coronavirus infection. J Virol. 2019;93(6):e01815-8.

13. Chen $Z$ et al. Function of HAb18G/ CD147 in invasion of host cells by severe acute respiratory syndrome coronavirus. J Infect Dis. 2005;191(5):755-60.

14. Wrapp D et al. Cryo-EM structure of the 2019-nCoV spike in the prefusion conformation. Science. 2020;367(6483):1260-3.

15. Wang $X$ et al. SARS-CoV-2 infects $T$ lymphocytes through its spike protein-mediated membrane fusion. Cell Mol Immunol. 2020:1-3.

16. Knoops $\mathrm{K}$ et al. SARS-coronavirus replication is supported by a reticulovesicular network of modified endoplasmic reticulum. PLoS Biol. 2008;6(9):e226. DOI:10.1371/journal. pbio.0060226.

17. Kindler E et al. Early endonucleasemediated evasion of RNA sensing ensures effi-cient coronavirus replication. PLoS Pathog. 2017;13(2):e1006195.

18. Chen $\mathrm{J}$ et al. Cellular immune responses to severe acute respiratory syndrome coronavirus (SARS-CoV) infection in senescent BALB/c mice: CD4+ T cells are important in control of SARS-CoV infection. J Virol. 2010;84(3):1289-301.

19. Ziegler T et al. Severe acute respiratory syndrome coronavirus fails to activate cytokine-mediated innate immune responses in cultured human monocyte-derived dendritic cells. J Virol. 2005;79(21):13800-5.

20. Wölfel R et al. Virological assessment of hospitalized patients with COVID-2019. Na-ture. 2020;581:465-9

21. Kamitani W et al. A two-pronged strategy to suppress host protein synthesis by SARS coronavirus Nsp1 protein. Nat Struct Mol Biol. 2009;16(11):1134-40
22. Yuan $L$ et al. p53 degradation by a coronavirus papain-like protease suppresses type I interferon signalling. J Biol Chem. 2015;290(5):3172-82

23. Li SW et al. SARS Coronavirus papain-like protease inhibits the TLR7 signalling pathway through removing Lys63-linked polyubiquitination of TRAF3 and TRAF6. Int J Mol Sci. 2016;17(5):E678.

24. Totura AL et al. Toll-like receptor 3 signalling via TRIF contributes to a protective innate immune response to severe acute respiratory syndrome coronavirus infec-tion. $\mathrm{mBio}$. 2015;6(3):e00638-15.

25. $\mathrm{Hu} X$ et al. Transmissible gastroenteritis virus papain-like protease 1 antagonizes production of interferon- $\beta$ through its deubiquitinase activity. Biomed Res Int. 2017;2017:7089091.

26. Zhao $\mathrm{J}$ et al. Evasion by stealth: inefficient immune activation underlies poor $\mathrm{T}$ cell response and severe disease in SARSCoV-infected mice. PLoS Pathog. 2009;5(10):e1000636.

27. Spiegel $M$ et al. Inhibition of beta interferon induction by severe acute respiratory syndrome coronavirus suggests a two-step model for activation of interferon regulatory factor 3. J Virol. 2005;79(4):2079-86.

28. Frieman $M$ et al. SARS coronavirus and innate immunity. Virus Res. 2008;133(1):101-2.

29. Cameron MJ et al. Human immunopathogenesis of severe acute respiratory syn-drome (SARS). Virus Res. 2008;133(1):13-9.

30. Cameron MJ et al. Interferonmediated immunopathological events are associated with atypical innate and adaptive immune responses in patients with severe acute respiratory syndrome. J Virol. 2007;81(16):8692-706

31. Cui $W$ et al. Expression of lymphocytes and lymphocyte subsets in patients with severe acute respiratory syndrome. Clin Infect Dis. 2003;37(6):857-9. 
32. Channappanavar $\mathrm{R}$ et al. Dysregulated Type I interferon and inflammatory monocyte-macrophage responses cause lethal pneumonia in SARS-CoV-infected mice. Cell Host Microbe. 2016;19(2):181-93.

33. Yang $L$ et al. Persistent memory CD4+ and CD8+ T-cell responses in recovered severe acute respiratory syndrome (SARS) patients to SARS coronavirus M antigen. J Gen Virol. 2007;88(Pt 10):2740-8

34. Cemerski S et al. The stimulatory potency of $T$ cell antigens is influenced by the formation of the immunological synapse. Immunity. 2007:26(3):345-55

35. Law HK et al. Chemokine upregulation in SARS-coronavirusinfected, monocyte-derived human dendritic cells. Blood. 2005;106(7):2366-74.

36. Otero DC et al. IFN- $\beta$ selectively inhibits IL-2 production through CREM-mediated chromatin remodeling. J Immunol. 2015;194(11):5120-8

37. Watanabe $M$ et al. Downmodulation of tumor suppressor $\mathrm{p} 53$ by $\mathrm{T}$ cell receptor signalling is critical for antigen-specific CD4(+) T cell responses. Immunity. 2014:40(5):681-91.

38. Zhao J et al. Airway memory CD4(+) T cells mediate protective immunity against emerging respiratory coronaviruses. Immunity. 2016;44(6):1379-91.

39. Kim KD et al. Adaptive immune cells temper initial innate responses. Nat Med. 2007:13(10):1248-52.

40. Channappanavar R, Perlman S Pathogenic human coronavirus infections: causes and consequences of cytokine storm and immunopathology. Semin Immunopathol. 2017;39(5):529-39.

41. Wang $X$ et al. SARS-CoV-2 infects $T$ lymphocytes through its spike protein-mediated membrane fusion. Cell Mol Immunol. 2020;DOI:10.1038/ s41423-020-0424-9.

42. Krähling $\vee$ et al. Severe acute respiratory syndrome coronavirus triggers apoptosis via protein kinase $\mathrm{R}$ but is resistant to its antiviral activity. J Virol. 2009;83(5):2298-309.

43. Hoang HD et al. Battling for ribosomes: translational control at the forefront of the antiviral response. $J$ Mol Biol. 2018;430(14):1965-92

44. García MA et al. The dsRNA protein kinase PKR: virus and cell control. Biochimie. 2007;89(6-7):799-811.

45. Gil J, Esteban M. Induction of apoptosis by the dsRNA-dependent protein kinase (PKR): mechanism of action. Apoptosis. 2000;5(2):107-14.

46. von Roretz C, Gallouzi IE. Protein kinase RNA/FADD/caspase-8 pathway mediates the proapoptotic activity of the RNA-binding protein human antigen R (HuR). J Biol Chem. 2010;285(22):16806-13.

47. Schulz $\mathrm{O}$ et al. Protein kinase $\mathrm{R}$ contributes to immunity against specific viruses by regulating interferon mRNA integrity. Cell Host Microbe. 2010;7(5):354-61.

48. Uetani K et al. Central role of doublestranded RNA-activated protein kinase in microbial induction of nitric oxide synthase. J Immunol. 2000;165(2):988-96.

49. Zamanian-Daryoush $\mathrm{M}$ et al. NF kappaB activation by doublestranded-RNA-activated protein kinase $(P K R)$ is mediated through NF-kappaB-inducing kinase and IkappaB kinase. Mol Cell Biol. 2000;20(4):1278-90.

50. Bonnet MC et al. PKR stimulates NF-kappaB irrespective of its kinase function by interacting with the IkappaB kinase complex. Mol Cell Biol. 2000;20(13):4532-42.

51. Kumar A et al. Double-stranded RNAdependent protein kinase activates transcription factor NF-kappa B by phosphorylating I kappa B. Proc Natl Acad Sci U S A. 1994;91(14):6288-92.

52. Goh KC et al. The protein kinase PKR is required for p38 MAPK activation and the innate immune response to bacterial endotoxin. EMBO J. 2000;19(16):4292-7.

53. Taghavi N, Samuel CE. Protein kinase PKR catalytic activity is required for the PKR-dependent activation of mitogen-activated protein kinases and amplification of interferon beta induction following virus infection. Virology. 2012;427(2):208-16.

54. Meusel TR et al. Protein kinase R regulates double-stranded RNA induction of TNF-alpha but not IL-1 beta mRNA in human epithelial cells. $\mathrm{J}$ Immunol. 2002;168(12):6429-35.

55. Gal-Ben-Ari S et al. PKR: a kinase to remember. Front Mol Neurosci. 2019;11:480.

56. Wang $\mathrm{H}$ et al. Double-stranded RNAdependent protein kinase deficiency protects the heart from systolic overload-induced congestive heart failure Circulation. 2014;129(13):1397-406.

57. $\mathrm{Ng}$ PC et al. Inflammatory cytokine profile in children with severe acute respiratory syndrome. Pediatrics. 2004;113(Part 1):e7-14.

58. He $Y$ et al. Mechanism and regulation of NLRP3 inflammasome activation. Trends Biochem Sci. 2016:41(12):1012-21.

59. Shi CS et al. SARS-coronavirus open reading frame-8b triggers intracellular stress pathways and activates NLRP3 inflammasomes. Cell Death Discov. 2019;5:101.

60. Siu KL et al. Severe acute respiratory syndrome coronavirus ORF3a protein activates the NLRP3 inflammasome by promoting TRAF3-dependent ubiquitination of ASC. FASEB J. 2019;33(8):8865-77.

61. Yim HC, Williams BR. Protein kinase $\mathrm{R}$ and the inflammasome. J Interferon Cytokine Res. 2014;34(6):447-54.

62. Yim $\mathrm{HC}$ et al. The kinase activity of PKR represses inflammasome activity. Cell Res. 2016;26(3):367-79.

63. Pouletty $M$ et al. Paediatric multisystem inflammatory syndrome temporally associated with SARS-CoV-2 mimicking Kawasaki disease (KawaCOVID-19): a multicentre cohort. Ann Rheum Dis. 2020;DOI:10.1136/ annrheumdis-2020-218814.

64. Alphonse MP et al. Inositol triphosphate 3-kinase $\mathrm{C}$ mediates inflammasome activation and treatment response in Kawasaki disease. J Immunol. 2016;197(9):3481-9.

65. Liu Z et al. Protein kinase R-like ER kinase and its role in endoplasmic reticulum stress-decided cell fate. Cell Death Dis. 2015;6:e1822

66. Wang $X Z$ et al. Signals from the stressed endoplasmic reticulum induce C/EBP-homologous protein (CHOP/GADD153). Mol Cell Biol. 1996;16(8):4273-80.

67. Kondratyev M et al. PERK-dependent compartmentalization of ERAD and unfolded protein response machineries during ER stress. Exp Cell Res. 2007;313(16):3395-407.

68. Minakshi $\mathrm{R}$ et al. The SARS Coronavirus $3 a$ protein causes endoplasmic reticulum stress and induces ligand-independent downregulation of the Type 1 interferon re-ceptor. PLoS One. 2009;4(12):e8342.

69. Paul D, Bartenschlager R. Architecture and biogenesis of plusstrand RNA virus replication factories. World J Virol. 2013;2(2):32-48.

70. Stertz $\mathrm{S}$ et al. The intracellular sites of early replication and budding of SARS-coronavirus. Virology. 2007;361(2):304-15.

71. Reggiori F et al. Coronaviruses hijack the LC3-I-positive EDEMosomes, ERderived vesicles exporting short-lived ERAD regulators, for replication. Cell Host Microbe. 2010;7(6):500-8.

72. Oostra M et al. Localization and membrane topology of coronavirus nonstructural protein 4: involvement of the early secretory pathway in replication. J Virol. 2007;81(22):12323-36.

73. van der Veen AG, Ploegh HL. Ubiquitin-like proteins. Annu Rev Biochem. 2012;81:323-57.

74. Byun $\mathrm{H}$ et al. ERAD and how viruses exploit it. Front Microbiol. 2014;5:330.

75. Hu J et al. Interleukin- 6 drives multiple myeloma progression by up-regulating of CD147/emmprin expression. Blood. 2016;128(22):5632. 
76. Arendt BK et al. Increased expression of extracellular matrix metalloproteinase inducer (CD147) in multiple myeloma: role in regulation of myeloma cell proliferation. Leukemia. 2012;26(10):2286-96.

77. Dai L et al. KSHV activation of VEGF secretion and invasion for endothelial cells is mediated through viral upregulation of emmprin-induced signal transduction. Int J Cancer. 2012;131(4):834-43.

78. Yurchenko $V$ et al. Dealing with the family: CD147 interactions with cyclophilins. Immunology. 2006;117(3):301-9.

79. Pfefferle $S$ et al. The SARScoronavirus-host interactome: identification of cyclophilins as target for pan-coronavirus inhibitors. PLoS Pathog. 2011;7(10):e1002331.

80. Fossum $\mathrm{S}$ et al. The MRC OX47 antigen is a member of the immunoglobulin super-family with an unusual transmembrane sequence. Eur J Immunol. 1991;21(3):671-9.

81. DeCastro R et al. Human keratinocytes express EMMPRIN, an extracellular matrix metalloproteinase inducer. J Invest Dermatol. 1996;106(6):1260-5.

82. Nehme $C L$ et al. Distribution of the integral plasma membrane glycoprotein CE9 (MRC OX-47) among rat tissues and its induction by diverse stimuli of metabolic activation. Biochem J. 1995:310(Part 2):693-8.

83. Seizer et al. EMMPRIN (CD147) is a novel receptor for platelet GPVI and mediates platelet rolling via GPVI-EMMPRIN interaction. Thromb Haemost. 2009;101(4):682-6.

84. Heinzmann D et al. CD147 is a novel interaction partner of integrin aMB2 mediating leukocyte and platelet adhesion. Biomolecules. 2020;10(4):E541.

85. Seizer $\mathrm{P}$ et al. Extracellular cyclophilin A activates platelets via EMMPRIN (CD147) and PI3K/Akt signalling, which promotes platelet adhesion and thrombus formation in vitro and in vivo. Arterioscler Thromb Vasc Biol. 2015;35(3):655-63.

86. Seizer $P$ et al. Cyclophilin A affects inflammation, virus elimination and myocardial fibrosis in coxsackievirus B3-induced myocarditis. J Mol Cell Cardiol. 2012;53(1):6-14

87. Elvers $M$ et al. Intracellular cyclophilin $A$ is an important $\mathrm{Ca}(2+)$ regulator in platelets and critically involved in arterial thrombus formation. Blood. 2012;120(6):1317-26.

88. Wu YP et al. Analysis of thrombotic factors in severe acute respiratory syndrome (SARS) patients. Thromb Haemost. 2006;96(1):100-1.

89. Jin R et al. Inhibition of CD147 (cluster of differentiation 147) ameliorates acute ischemic stroke in mice by reducing thromboinflammation. Stroke. 2017;48(12):3356-65.

90. Wang $\mathrm{H}$ et al. SARS coronavirus entry into host cells through a nove clathrin- and caveolae-independent endocytic pathway. Cell Res. 2008;18(2):290-301.

91. Haga $S$ et al. Modulation of TNFalpha-converting enzyme by the spike protein of SARS-CoV and ACE2 induces TNF-alpha production and facilitates viral entry. Proc Natl Acad Sci U S A. 2008;105(22):7809-14.

92. Xie $X$ et al. Age- and gender-related difference of ACE2 expression in rat lung. Life Sci. 2006;78(19):2166-71.

93. Guan WJ et al. Comorbidity and its impact on 1590 patients with COVID-19 in China: a nationwide analysis. Eur Respir J. 2020;55(5):2000547.

94. Russo $P$ et al. COVID-19 and smoking: is nicotine the hidden link? Eur Respir J. 2020;55(6):2001116.

95. Liu $Y$ et al. Clinical and biochemical indexes from 2019-nCoV infected patients linked to viral loads and lung injury. Sci China Life Sci. 2020;63:364-74

96. Aguilera $\mathrm{G}$ et al. The renin angiotensin system and the stress response. Ann N Y Acad Sci. 1995;771(1):173-86.

97. Kuba K et al. A crucial role of angiotensin converting enzyme 2 (ACE2) in SARS coronavirus-induced lung injury. Nat Med. 2005;11(8):875-9.

98. Imai $Y$ et al. Angiotensin-converting enzyme 2 protects from severe acute lung failure. Nature. 2005:436(7047):112-6.

99. Nguyen Dinh Cat A et al. Angiotensin II, NADPH oxidase, and redox signalling in the vasculature. Antioxid Redox Signal. 2013;19(10):1110-20.

100. Li G et al. NADPH oxidase links endoplasmic reticulum stress, oxidative stress, and PKR activation to induce apoptosis. J Cell Biol. 2010;191(6):1113-25.

101. Gheblawi M et al. Angiotensinconverting enzyme 2: SARS-CoV-2 receptor and regulator of the reninangiotensin system: celebrating the $20^{\text {th }}$ anniversary of the discovery of ACE2. Circ Res. 2020;126(10):1456-74

102. Kikuchi G et al. Heme oxygenase and heme degradation. Biochem Biophys Res Commun. 2005;338(1):558-67.

103. Espinoza JA et al. Modulation of antiviral immunity by heme oxygenase-1. Am J Pathol. 2017:187(3):487-93.

104. Konrad FM et al. Tissue heme oxygenase- 1 exerts anti-inflammatory effects on LPS-induced pulmonary inflammation. Mucosal Immunol. 2016;9(1):98-111.
105. Chauveau $\mathrm{C}$ et al. Heme oxygenaseexpression inhibits dendritic cell maturation and proinflammatory function but conserves IL-10 expression. Blood. 2005;106(5):1694-702

106. Spiegel $M$ et al. Interaction of severe acute respiratory syndromeassociated coro-navirus with dendritic cells. J Gen Virol. 2006;87(Part 7):1953-60.

107. Vijay $R$ et al. Critical role of phospholipase A2 group IID in agerelated susceptibility to severe acute respiratory syndrome-CoV infection. J Exp Med. 2015;212(11):1851-68.

108. Tzima S et al. Myeloid heme oxygenase-1 regulates innate immunity and autoimmunity by modulating IFN-beta production. J Exp Med. 2009;206(5):1167-79.

109. Matthews $\mathrm{K}$ et al. The SARS coronavirus papain like protease can nhibit IRF3 at a post activation step that requires deubiquitination activity Virol J. 2014;11:209.

110. Taira $\mathrm{J}$ et al. Caveolin-1 is a competitive inhibitor of heme oxygenase-1 ( $\mathrm{HO}-1)$ with heme: identification of a minimum sequence in caveolin-1 for binding to $\mathrm{HO}-1$. Biochemistry. 2011;50(32):6824-31.

111. Zhang JS et al. Gastrodin suppresses BACE1 expression under oxidative stress con-dition via inhibition of the PKR/elF2a pathway in Alzheimer's disease. Neurosci-ence. 2016;325:1-9.

112. Liu $Y$ et al. A review on central nervous system effects of gastrodin. Front Pharmacol. 2018:9:24:DOl:10.3389/ fphar.2018.00024.

113. Weintraub S et al. Design and synthesis of novel protein kinase $\mathrm{R}(\mathrm{PKR})$ inhibitors. Mol Divers. 2016;20(4):805-19.

114. Wang $D$ et al. Auto-phosphorylation represses protein kinase $\mathrm{R}$ activity. Sci Rep. 2017;7:44340.

115. Mata M et al. N-acetyl-L-cysteine (NAC) inhibit mucin synthesis and pro-inflammatory mediators in alveolar type II epithelial cells infected with influenza virus $A$ and $B$ and with respiratory syncytial virus (RSV). Biochem Pharmacol. 2011;82(5):548-55

116. Chu WM et al. JNK2 and IKKbeta are required for activating the innate response to viral infection. Immunity. 1999;11(6):721-31.

117. Silva $A M$ et al. Protein kinase $R(P K R)$ interacts with and activates mitogenactivated protein kinase kinase 6 (MKK6) in response to doublestranded RNA stimulation. J Bio Chem. 2004;279(36):37670-6.

118. Donadelli $\mathrm{R}$ et al. Protein traffic activates NF-kB gene signalling and promotes MCP-1-dependent 
interstitial inflammation. Am J Kidney Dis. 2000;36(6):1226-41.

119. Fu ZG et al. A novel small-molecule compound targeting CD147

inhibits the motility and invasion

of hepatocellular carcinoma cells.

Oncotarget. 2016;7(8):9429-47.
120. Pahk K et al. A novel CD147 inhibitor, SP-8356, reduces neointimal hyperplasia and arterial stiffness in a rat model of partial carotid artery ligation. J TransI Med. 2019;17(1):274.

121. U.S. Food and Drug Administration (FDA). Meplazumab: search orphan drug desig-nations and approvals. 2020. Available at: https://www. accessdata.fda.gov/scripts/ opdlisting/oopd/detailedlndex. cfm?cfgridkey $=676218$. Last accessed: 7 October 2020 